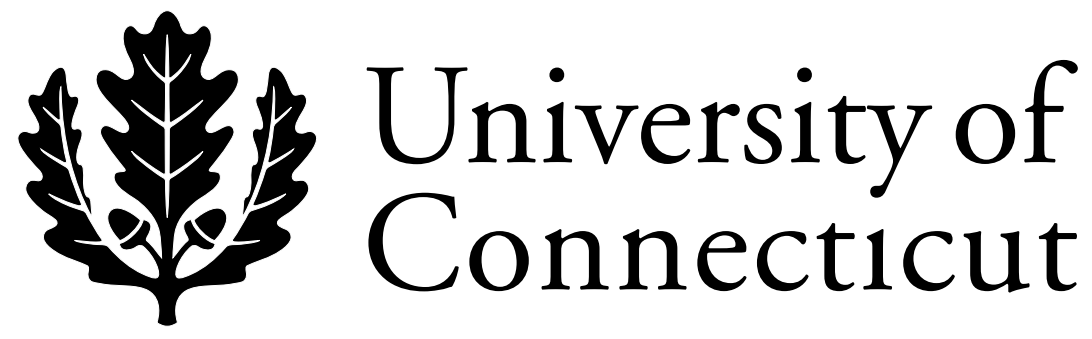

Department of Economics Working Paper Series

\title{
Characterizing Paretian Preferences
}

Vicki Knoblauch

University of Connecticut

Working Paper 2003-30

August 2003

341 Mansfield Road, Unit 1063

Storrs, CT 06269-1063

Phone: (860) 486-3022

Fax: (860) 486-4463

http://www.econ.uconn.edu/ 


\begin{abstract}
A characterization of a property of binary relations is of type $M$ if it can be stated in terms of ordered $M$-tuples of alternatives. A characterization of finite type provides an easy test of whether preferences over a large set of alternatives possesses the property characterized. Unfortunately, there is no characterization of finite type for Pareto representability in $\mathcal{R}^{2}$. A partial result along the same lines is obtained for Pareto representability in $\mathcal{R}^{k}, k>2$.
\end{abstract}

Journal of Economic Literature Classification: D11, D70

Keywords: preferences, Pareto order, voting 


\section{Introduction: Characterizations of Finite Type.}

By a theorem of Debreu (1964) a binary relation on a finite set can be represented by a utility function if and only if it is asymmetric and its associated weak relation ${ }^{1}$ is transitive. This characterization will be called a type 3 characterization, since it can be stated in terms of ordered triples of alternatives: $\succ$ on a finite set $X$ has a utility representation if and only if for all $a, b, c \in X a \succ b$ implies $a \succsim b$, and $a \succsim b \succsim c$ implies $a \succsim c$.

Characterizations of finite type are useful, since the conditions comprising a characterization of finite type can be checked in polynomial time. This means that if $C$ is a characterization of finite type of some property of binary relations (such as utility representability), then there is a polynomial $P$ such that $P(N)$ steps are sufficient to determine whether a binary relation on a set of $N$ alternatives satisfies the conditions of $C$. For example consider the type 3 characterization of utility representability given above. When this characterization is used to determine whether a binary relation on a set of $N$ alternatives can be represented by a utility function, it may be necessary to consider all $N^{3}$ ordered triples of alternatives. For each ordered triple $\langle a, b, c\rangle$, the ordered pairs comprising $\succ$ (there are at most $N^{2}$ ) are examined to determine whether $a \succ b$, whether $a \succsim b$, whether $b \succsim c$ and whether $a \succsim c$. Then two steps are required to check whether $a \succ b$ implies $a \succsim b$ and whether $a \succsim b \succsim c$ implies $a \succsim c$. Therefore at most $N^{3}\left(N^{2}+2\right)=N^{5}+2 N^{3}$ steps are needed to determine whether a given binary relation on a set of $N$ alternatives satisfies the conditions characterizing utility representability.

The existence of such a polynomial is desirable, since $P(N)$ grows with $N$ much more slowly than, say, $2^{N}$ so that characterizations of finite type are useful for testing binary relations on relatively large sets of alternatives.

For a given $k$, is there a characterization of finite type for Pareto representability in $\Re^{k}$ of preferences over finite sets? ${ }^{2}$ There are two facts that when taken together suggest that the answer might be yes. First, Debreu's characterization of utility representability mentioned above consists of three conditions; asymmetry, separability and transitivity

\footnotetext{
1 For a binary relation $\succ$ on $X$, the associated weak relation $\succsim$ on $X$ is defined by $x \succsim y$ if $\operatorname{not}(y \succ x)$.

2 A Pareto representation in $\Re^{k}$ for a binary relation $\succ$ on $X$ is a function $v: X \rightarrow \Re^{k}$ such that $x \succ y$ if and only if $v(x)>v(y)$, where $v(x)>v(y)$ if $v_{i}(x) \geq v_{i}(y)$ for $1 \leq i \leq k$ and $v(x) \neq v(y)$.
} 
of the associated weak relation. Since every binary relation on a finite set is separable, for binary relations on finite sets the separability condition can be dropped, leaving a characterization shown above to be of finite type.

Second, Sprumont's (2001) characterization of Pareto representability in $\Re^{2}$ consists of three conditions he calls intermediateness conditions plus a continuity condition and a condition he calls richness. By analogy with Debreu's characterization of utility representability, it might be possible, when considering only preferences over finite sets, to drop the continuity and richness conditions of Sprumont's characterization, leaving the intermediateness conditions, which are of finite type.

Unfortunately, as will be proven below, Pareto representability in $\Re^{2}$ has no characterization of finite type even when interest is restricted to binary relations on finite sets. It should be mentioned here that the definition of Pareto representability in $\Re^{2}$ is itself a characterization of Pareto representability in $\Re^{2}$, but not a characterization of finite type, since although only ordered pairs are mentioned explicitly in that definition the requirement of the existence of a function $v: X \rightarrow \Re^{2}$ cannot be reformulated in terms of ordered $M$-tuples of alternatives for a fixed $M$.

However, to say that a property of binary relations on finite sets has no characterization of finite type is not to say that that property has no useful characterizations. There may be a characterization that is useful when $N=|X|$ is small, but not so small that it is easy to check directly whether the property holds; or there may be a characterization that is useful for some binary relations even when $N$ is large. For example, it is well-known that a binary relation $\succ$ on a finite set can be extended to a linear order ${ }^{3}$ if and only if it contains no cycle $x^{1} \succ x^{2} \succ \ldots \succ x^{m} \succ x^{1}$. This characterization is not of finite type since it can't be stated in terms of $M$-tuples of alternatives for a fixed integer $M$; its statement uses $m$-tuples where $m$ is restricted only by $1 \leq m \leq N=|X|$. However, the characterization is useful; if a binary relation is presented as a directed graph it is sometimes easy to determine by inspection whether the graph contains a directed cycle.

\footnotetext{
3 A linear order is an asymmetric, transitive binary relation whose associated weak relation is antisymmetric $(x \succsim y \succsim x$ implies $x=y)$. To say $\succ$ can be extended to $\succ^{*}$ means for $x, y \in X, x \succ y$ implies $x \succ^{*} y$.
} 
Returning to the question of Pareto representability, given that Pareto representability in $\Re^{2}$ has no characterization of finite type, it is reasonable to conjecture that the same is true of Pareto representability in $\Re^{k}$ for $k>2$. Toward a proof of this, it is established below that for $k \geq 2$ every characterization of Pareto representability in $\Re^{k}$ is of type $2 k+2$ or greater.

Past studies concerned with Pareto representation include the aforementioned paper of Sprumont (2001); Dushnik and Miller's (1941) characterizations of Pareto representability in $\Re^{2}$; and Aizerman and Aleskerov's (1995) algorithm for finding, for a given asymmetric, transitive binary relation, the minimal $k$ such that the relation has a Pareto representation in $\Re^{k}$.

These and other authors have concerned themselves with the Pareto relation in part because of its use in group decision making, in the form of unanimous voting or decisions governed by veto. For example, permanent members of the U. N. Security Council possess veto power over resolutions before that body; some fraternities, sororities and other clubs grant their members the power to blackball aspiring members; many academics have spoken or at least heard words like these: "Yes we can move the test to Thursday, but only if everyone in the class agrees;" and finally corresponding to the case $k=2$ above, the consuls of ancient Rome served in pairs and could only act in tandem.

A simple characterization of Pareto representability in $\Re^{k}$ would therefore be interesting because, among other things, it would allow one to determine easily whether a given set of preferences could have been generated by unanimous voting by a committee of $k$ members, each governed by a utility function.

The rest of the paper is organized as follows. The main results are stated in Section 2. Section 3 introduces an example used in Section 4 in the proofs of the main results.

\section{Preliminaries and Two Theorems.}

A binary relation $\succ$ on a set $X$ is a subset of $X \times X$. For convenience $<x, y>\in \succ$ will be written $x \succ y$. Associated with each $\succ$ on $X$, there are binary relations $\succsim$ and $\sim$ on $X$ defined by $x \succsim y$ if $\operatorname{not}(y \succ x)$ and $x \sim y$ if $x \succsim y \succsim x$. A binary relation $\succ$ on $X$ is asymmetric if for all $x, y \in X x \succ y$ implies $x \succsim y$ and transitive if for all $x, y \in X x \succ y \succ z$ 
implies $x \succ z$. For $\succ$ on $X$ and $Y \subseteq X$, the restriction $\left.\succ\right|_{Y}$ of $\succ$ is the binary relation on $Y$ defined by $\left.\succ\right|_{Y}=\succ \cap(Y \times Y)$. If $\succ$ on $X$ and $x \in X$ let $W(x)=\{z \in X: x \succ z\}$.

The Pareto relation $>$ on $\Re^{k}$ is defined by $r>s$ if $r_{i} \geq s_{i}$ for $i \in\{1,2, \ldots, k\}$ and $r \neq s$. A Pareto representation in $\Re^{k}$ for $\succ$ on $X$ is a function $v: X \rightarrow \Re^{k}$ such that for all $x, y \in X x \succ y$ if and only if $v(x)>v(y)$.

It is often useful to characterize-simply-all binary relations that have a certain interesting property. A characterization of a property $Q$ is a statement of the form, "A binary relation $\succ$ on $X$ satisfies property $Q$ if and only if for every ordered $m$-tuple $<x^{1}, x^{2}, \ldots, x^{m}>$ of elements of $X, \ldots$," where the ellipses represent conditions in the form of an expression involving $\succ, x^{1}, x^{2}, \ldots, x^{m}$ and various logical, set theoretic and arithmetic symbols. A characterization is said to be of type $M$, for positive integer $M$, if the conditions begin with the proviso that only $m$-tuples with $m \leq M$ need to be considered.

Section 1 contained an example of a property with no characterization of finite type (extendability to a linear order) and an example of a property with a characterization of type 3 (representability by a utility function). The demonstration in Section 1 that one can determine in at most $N^{5}+2 N^{3}$ steps whether a binary relation on a set of $N$ alternatives satisfies Debreu's characterization of utility representability can be generalized to establish that in general a characterization of finite type can be checked in polynomial time.

We can now state the main results which concern characterizations of finite type for Pareto representability of binary relations on finite sets.

Theorem 1. There is no characterization of finite type for Pareto representability in $\Re^{2}$.

Theorem 2. If $k>2$, every characterization of Pareto representability in $\Re^{k}$ is of type $2 k+2$ or greater .

\section{An Example.}

The following example presents binary relations that are almost (Propositions 2 and 3) but not quite (Proposition 1) Pareto representable in $\Re^{k}$. 
Example 1. For $n>k \geq 2$ let $X^{n}=\left\{x^{1}, x^{2}, \ldots, x^{n}, y^{1}, y^{2}, \ldots y^{n}\right\}$ and define $\succ^{k}$ on $X^{n}$ by $x^{i} \succ^{k} y^{j}$ if there exists $l \in\{1,2, \ldots, k\}$ such that $j=i \oplus l$, where $\oplus$ indicates addition $\bmod n$ on $\{1,2, \ldots, n\}$; that is,

$$
i \oplus l= \begin{cases}i+l & \text { if } i+l \leq n \\ i+l-n & \text { if } i+l>n\end{cases}
$$

It will be seen in Propositions 1, 2 and 3 below that if $n>k=2$ or if $n-1=$ $k \geq 2$, then $\succ^{k}$ on $X^{n}$ of Example 1 has no Pareto representation in $\Re^{k}$, but under the same conditions on $k$ and $n$, if $S$ is any proper subset of $X^{n}$, then $\left.\succ^{k}\right|_{S}$ has a Pareto representation in $\Re^{k}$.

Proposition 1. If $n>k=2$, or if $n-1=k \geq 2$, then $\succ^{k}$ on $X^{n}$ is not Pareto representable in $\Re^{k}$.

Proof. Suppose $v: X^{n} \rightarrow \Re^{k}$ is a Pareto representation for $\succ^{k}$. For each $i \in\{1,2, \ldots, k\}$ let $f_{i}$ be a permutation of $\{1,2, \ldots, n\}$ such that $v_{i}\left(y^{f_{i}(1)}\right) \geq v_{i}\left(y^{f_{i}(2)}\right) \geq \ldots \geq v_{i}\left(y^{f_{i}(n)}\right)$.

If $n-1=k \geq 2$, there exists $j^{o} \in\{1,2, \ldots n\}$ such that for all $i \in\{1,2, \ldots, k\}, j^{o} \neq$ $f_{i}(1)$. Therefore for each $i v_{i}\left(x^{j^{0}}\right) \geq v_{i}\left(y^{f_{i}(1)}\right) \geq v_{i}\left(y^{j^{0}}\right)$. Also, since $W\left(x^{j^{o}}\right) \neq W\left(y^{j^{o}}\right)$, $v\left(x^{j^{o}}\right) \neq v\left(y^{j^{o}}\right)$. Since $v\left(x^{j^{o}}\right) \neq v\left(y^{j^{o}}\right)$ and $v_{i}\left(x^{j^{o}}\right) \geq v_{i}\left(y^{j^{o}}\right)$ for all $i, v\left(x^{j^{o}}\right)>v\left(y^{j^{o}}\right)$. Therefore $x^{j^{o}} \succ^{k} y^{j^{o}}$, contradicting the definition of $\succ^{k}$.

Next, suppose $n>k=2$. Since $\sum_{i=1}^{2} \sum_{j=1}^{n} f_{i}(j)=n(n+1)$, for some $j^{o} \in$ $\{1,2, \ldots, n\}, \sum_{i=1}^{2} f_{i}\left(j^{0}\right) \geq n+1, f_{1}\left(j^{o}\right) \neq 1$ and $f_{2}\left(j^{o}\right) \neq 1$. Therefore, $\mid\left\{j \neq j^{o}: v_{1}\left(y^{j}\right) \geq\right.$ $\left.v_{1}\left(y^{j^{0}}\right)\right\}|+|\left\{j \neq j^{o}: v_{2}\left(y^{j}\right) \geq v_{2}\left(y^{j^{0}}\right)\right\} \mid \geq n-1$. Therefore there exist $j^{1} \in\{j \neq$ $\left.j^{o}: v_{1}\left(y^{j}\right) \geq v_{1}\left(y^{j^{o}}\right)\right\}$ and $j^{2} \in\left\{j \neq j^{o}: v_{2}\left(y^{j}\right) \geq v_{2}\left(y^{j^{o}}\right)\right\}$ such that $j^{2}=j^{1}$ or $j^{2}=j^{1} \oplus 1$ or $j^{1}=j^{2} \oplus 1$. Then there exists $j^{3}$ such that $x^{j^{3}} \succ^{2} y^{j^{1}}, x^{j^{3}} \succ^{2} y^{j^{2}}$ and $y^{j^{o}} \succsim^{2} x^{j^{3}}$, so that $v_{1}\left(x^{j^{3}}\right) \geq v_{1}\left(y^{j^{1}}\right) \geq v_{1}\left(y^{j^{o}}\right)$ and $v_{2}\left(x^{j^{3}}\right) \geq v_{2}\left(y^{j^{2}}\right) \geq v_{2}\left(y^{j^{o}}\right)$. Also $v\left(x^{j^{3}}\right) \neq v\left(y^{j^{0}}\right)$ since $W\left(x^{j^{3}}\right) \neq W\left(y^{j^{o}}\right)$. Therefore, $v\left(x^{j^{3}}\right)>v\left(y^{j^{0}}\right)$ so that $x^{j^{3}} \succ^{2} y^{j^{0}}$ contradicting our choice of $j^{3}$. 
Proposition 2. If $n>2$, and $S$ is a proper subset of $X^{n}$, then $\left.\succ^{2}\right|_{S}$ is Pareto representable in $\Re^{2}$.

Proof. Define $v: X^{n} \rightarrow \Re^{2}$ by $v\left(x^{i}\right)=<i, n-i>, v\left(y^{j}\right)=<j-2, n-j+1>$ if $j \neq 1$ and $v\left(y^{1}\right)=<n-1,0>$.

If $x^{i}, x^{j} \in X$, then $v\left(x^{i}\right) \geq v\left(x^{j}\right)$ since $i>j$ implies $v_{1}\left(x^{i}\right)>v_{1}\left(x^{j}\right) ; i<j$ implies $v_{2}\left(x^{i}\right)>v_{2}\left(x^{j}\right)$; and $i=j$ implies $v\left(x^{i}\right)=v\left(x^{j}\right)$. Similarly, if $x^{i}, y^{i}, y^{j} \in X^{n}$, then $v\left(y^{i}\right) \geq v\left(y^{j}\right)$ and $v\left(x^{i}\right) \geq v\left(y^{j}\right)$.

Next, $v\left(x^{i}\right)>v\left(y^{j}\right)$ if and only if $v_{1}\left(x^{i}\right) \geq v_{1}\left(y^{j}\right), v_{2}\left(x^{i}\right) \geq v_{2}\left(y^{j}\right)$ and $v\left(x^{i}\right) \neq v\left(y^{j}\right)$ if and only if $i \geq j-2$ and $n-i \geq n-j+1$ or $y=1$ and $i=n-1$ or $n$ if and only if $j=i+1$ or $i+2$, or $j=1$ and $i=n-1$ or $n$ if and only if $j=i \oplus 1$ or $j=i \oplus 2$, and $i \neq n$ or $j \neq 2$. Therefore for $x^{i}, y^{j} \in X^{n}-\left\{x^{n}\right\}$ or $x^{i}, y^{j} \in X^{n}-\left\{y^{2}\right\}, v\left(x^{i}\right)>v\left(y^{j}\right)$ if and only if $j=i \oplus 1$ or $j=i \oplus 2$ if and only if $x^{i} \succ^{2} y^{j}$.

Summarizing the conclusions of the previous two paragraphs, $\left.v\right|_{X^{n}-\left\{x^{n}\right\}}$ is a Pareto representation for $\left.\succ^{2}\right|_{X^{n}-\left\{x^{n}\right\}}$ and $\left.v\right|_{X^{n}-\left\{y^{2}\right\}}$ is a Pareto representation for $\left.\succ^{2}\right|_{X^{n}-\left\{y^{2}\right\}}$.

The Pareto representability in $\Re^{2}$ of $\left.\succ^{2}\right|_{X^{n}-\left\{x^{n}\right\}}$ and the existence for each $j \in$ $\{1,2, \ldots, n\}$ of a one-to-one, onto, order preserving function $f: X^{n}-\left\{x^{n}\right\} \rightarrow X^{n}-\left\{x^{j}\right\}$ together imply the Pareto representability in $\Re^{2}$ of $\left.\succ^{2}\right|_{X^{n}-\left\{x^{j}\right\}}$. Here is that $f$ : for $i \in\{1,2, \ldots, n-1\} f\left(x^{i}\right)=x^{i \oplus j}$, and for $i \in\{1,2, \ldots, n\} f\left(y^{i}\right)=y^{i \oplus j}$.

Similarly, $\left.\succ^{2}\right|_{X^{n}-\left\{y^{j}\right\}}$ is Pareto representable in $\Re^{2}$ for every $j \in\{1,2, \ldots, n\}$.

Finally if $S$ is a proper subset of $X$, then for some $j \in\{1,2, \ldots, n\} S \subseteq X-\left\{x^{j}\right\}$ or $S \subseteq X-\left\{y^{j}\right\}$, Therefore $\left.\succ^{2}\right|_{S}$ is Pareto representable in $\Re^{2}$.

Proposition 3. If $n-1=k \geq 2$, and $S$ is a proper subset of $X^{n}=X^{k+1}$, then $\left.\succ^{k}\right|_{S}$ is Pareto representable in $\Re^{k}$. 
Proof. Let $\oplus^{k}$ indicate addition $\bmod k$ on $\{1,2 \ldots, k\}$ and $\oplus^{n}$ indicate addition $\bmod n$ on $\{1,2 \ldots, n\}$. Construct $v: X^{k+1}-\left\{y^{k+1}\right\} \rightarrow \Re^{k}$ such that for each $i \in\{1,2, \ldots, k\}$

$$
\begin{gathered}
v_{i}\left(x^{i}\right)>v_{i}\left(x^{i \oplus^{k} 1}\right)>\ldots>v_{i}\left(x^{i \oplus^{k}(k-2)}\right)>v_{i}\left(x^{k+1}\right)>v_{i}\left(y^{i \oplus^{k}(k-1)}\right)> \\
v_{i}\left(x^{i \oplus^{k}(k-1)}\right)>v_{i}\left(y^{i}\right)>v_{i}\left(y^{i \oplus^{k} 1}\right)>\ldots>v_{i}\left(y^{i \oplus^{k}(k-2)}\right)
\end{gathered}
$$

Allowing $i$ to vary over $\{1,2, \ldots, k\}$ it follows from (1) that for all $i, j \in\{1,2, \ldots, k+1\}$ $v\left(x^{i}\right) \geq v\left(x^{j}\right) ;$ for all $i, j \in\{1,2, \ldots, k\} v\left(y^{i}\right) \geq v\left(y^{j}\right)$; and for $i \in\{1,2, \ldots, k+1\}$, $j \in\{1,2, \ldots, k\} v\left(x^{i}\right)>v\left(y^{j}\right)$ if and only if $i \neq j$.

Summarizing the above, for $w, z \in X^{k+1}-\left\{y^{k+1}\right\}, v(w)>v(z)$ if and only if $w=$ $x^{i}, z=y^{j}$ and $i \neq j$ if and only if $w=x^{i}, z=y^{j}$ and $j=i \oplus^{n} l$ for some $l \in\{1,2, \ldots, k\}$ if and only if $w \succ^{k} z$. Therefore $v$ is a Pareto representation for $\left.\succ^{k}\right|_{X^{k+1}-\left\{y^{k+1}\right\}}$.

Arguing from the symmetry of $\succ^{k},\left.\succ^{k}\right|_{X^{k+1}-\left\{y^{j}\right\}}$ is Pareto representable in $\Re^{k}$ for every $j \in\{1,2, \ldots, k+1\}$.

By an identical proof, $\left.\succ^{k}\right|_{X^{k+1}-\left\{x^{j}\right\}}$ is Pareto representable in $\Re^{k}$.

If $S$ is a proper subset of $X^{k+1}$, then for some $j, S \subseteq X^{k+1}-\left\{x^{j}\right\}$ or $S \subseteq X^{k+1}-\left\{y^{j}\right\}$. Therefore $\left.\succ\right|_{S}$ is Pareto representable in $\Re^{k}$.

These three propositions supply easy proofs of Theorems 1 and 2 .

\section{Proofs of Theorems 1 and 2.}

Proof of Theorem 1. Suppose $C$ is a characterization of type $M$ for Pareto representability in $\Re^{2}$. Choose $n>2$ such that $2 n>M$. Let $\succ^{2}$ on $X^{n}$ be as in Example 1. Since by Proposition 1 with $n>k=2, \succ^{2}$ is not Pareto representable in $\Re^{2}$ and since $C$ is a characterization of type $M$ of Pareto representability in $\Re^{2}$, there exist $z^{1}, z^{2}, \ldots, z^{M} \in X^{n}$ that do not satisfy the conditions comprising $C$. Therefore $\left.\succ^{2}\right|_{\left\{z^{1}, z^{2}, \ldots, z^{M}\right\}}$ is not Pareto representable in $\Re^{2}$, contradicting Proposition 2 , which applies since $\left|\left\{z^{1}, z^{2}, \ldots, z^{M}\right\}\right| \leq$ $M<2 n=\left|X^{n}\right|$ so that $\left\{z^{1}, z^{2}, \ldots, z^{M}\right\}$ is a proper subset of $X^{n}$. 
Proof of Theorem 2. Suppose $C$ is a characterization of type $2 k+1$ for Pareto representability in $\Re^{k}$. Let $\succ^{k}$ on $X^{k+1}$ be as in Example 1. Since by Proposition 1 with $n-1=k \geq 2$, $\succ^{k}$ is not Pareto representable in $\Re^{k}$ and since $C$ is a characterization of type $2 k+1$, there exist $z^{1}, z^{2}, \ldots, z^{2 k+1} \in X^{k+1}$ that do not satisfy the conditions comprising $C$. Therefore $\left.\succ^{k}\right|_{\left\{z^{1}, z^{2}, \ldots, z^{2 k+1}\right\}}$ is not Pareto representable in $\Re^{k}$, contradicting Proposition 3, which applies since $\left|\left\{z^{1}, z^{2}, \ldots, z^{2 k+1}\right\}\right| \leq 2 k+1<2 k+2=\left|X^{k+1}\right|$ so that $\left\{z^{1}, z^{2}, \ldots, z^{2 k+1}\right\}$ is a proper subset of $X^{n}$.

\section{Concluding Remarks.}

An examination of the proof of Theorem 1 reveals that in order to prove that there is no characterization of finite type for Pareto representability in $\Re^{k}$, it would be sufficient to construct an example of $\succ$ on $X$ with $|X|$ arbitrarily large such that $\succ$ is not Pareto representable in $\Re^{k}$, but $\left.\succ\right|_{S}$ is Pareto representable in $\Re^{k}$ if $S$ is any proper subset of $X$. 


\section{References}

1. M. Aizerman and F. Aleskerov, "Theory of Choice," North-Holland, Amsterdam, 1995.

2. G. Debreu, Continuity properties of paretian utility, International Economic Review 5 (1964), 285-293.

3. B. Dushnik and W. Miller, Partially ordered sets, American Journal of Mathematics 63 (1941), 600-610.

4. Y. Sprumont, Paretian quasi-orders: the regular two-agent case, Journal of Economic Theory 101 (2001), 437-456. 